\title{
On Weak and Strong Convergence Theorems for a Finite Family of Nonself $I$-asymptotically Nonexpansive Mappings
}

\author{
Birol GÜndüZ And Sezgin AkBulut
}

\begin{abstract}
We prove the weak and strong convergence of $\mathrm{S}$ iterative scheme to a common fixed point of a family of nonself asymptotically $I$-nonexpansive mappings $\left\{T_{i}\right\}_{i}^{N}$ and a family of nonself asymptotically nonexpansive mappings $\left\{I_{i}\right\}_{i}^{N}$, defined on a nonempty closed convex subset of a Banach space. Our scheme converges faster than Mann and Ishikawa iteration for contractions. Our weak convergence theorem is proved under more general setup of space as different from weak convergence theorems proved in previously.
\end{abstract}

\section{INTRODUCTION}

Let $X$ be a real normed linear space and $K$ be a nonempty subset of $X$. Let $T$ be a self-mapping of $K$.

(1) $T$ is said to be nonexpansive if $\|T x-T y\| \leq\|x-y\|$ holds for all $x, y \in K$.

(2) $T$ is said to be asymptotically nonexpansive if there exists a sequence $\left\{k_{n}\right\} \in[0, \infty)$, with $\lim _{n \rightarrow \infty} k_{n}=0$, such that $\left\|T^{n} x-T^{n} y\right\| \leq$ $\left(1+k_{n}\right)\|x-y\|$ for all $x, y \in K$ and $n \geq 1$.

(3) $T$ is called uniformly $L$-Lipschitzian if there exists constant $L>0$ such that $\left\|T^{n} x-T^{n} y\right\| \leq L\|x-y\|$ for all $x, y \in K$ and positive integer $n \geq 1$.

Being an important generalization of nonexpansive mapping, the concept of asymptotically nonexpansive self-mapping was proposed by Goebel and Kirk [1] in 1972. In [1], it was proved that if $X$ is uniformly convex, and $K$ is a bounded closed and convex subset of $X$, then every asymptotically nonexpansive self mapping has a fixed point.

Iterative techniques for asymptotically nonexpansive self-mapping in Banach spaces including Mann type and Ishikawa type iterations processes have been studied extensively by various authors. However, if the domain of $T$,

2010 Mathematics Subject Classification. Primary: 47H10; Secondary: 54H25.

Key words and phrases. Nonself asymptotically $I$-nonexpansive mappings; Iterative scheme; Kadec-Klee property; Condition (B); Common fixed point. 
$D(T)$, is a proper subset of $E$ (and this is the case in several applications), and $T$ maps $D(T)$ into $E$, then the iteration processes of Mann type and Ishikawa type and their modifications introduced may fail to be well defined.

A subset $K$ of $X$ is said to be a retract of $X$ if there exists a continuous map $P: X \rightarrow K$ such that $P x=x$, for all $x \in K$. Every closed convex subset of a uniformly convex Banach space is a retract. A map $P: X \rightarrow K$ is said to be a retraction if $P^{2}=P$. It follows that, if a map $P$ is a retraction, then $P y=y$ for all $y$ in the range of $P$.

In 2003, Chidume, Ofoedu and Zegeye [15] further generalized the concept of asymptotically nonexpansive self-mapping, and proposed the concept of nonself asymptotically nonexpansive mapping, which is defined as follows:

Definition $1.1([15])$. Let $K$ be a nonempty subset of a real normed space $X$ and $P: X \rightarrow K$ be a nonexpansive retraction of $X$ onto $K$.

(1) Mapping $T: K \rightarrow X$ is called asymptotically nonexpansive if there exists a sequence $\left\{k_{n}\right\} \subset[0, \infty)$ with $k_{n} \rightarrow 0$ as $n \rightarrow \infty$ such that for any positive integer $n$ and all $x, y \in K$

$$
\left\|T(P T)^{n-1} x-T(P T)^{n-1} y\right\| \leq\left(1+k_{n}\right)\|x-y\| .
$$

(2) Non-self mapping $T: K \rightarrow X$ is said to be uniformly L-Lipschitzian if there exists a constant $L>0$ such that for all $x, y \in K$

$$
\left\|T(P T)^{n-1} x-T(P T)^{n-1} y\right\| \leq L\|x-y\| .
$$

Note that if $P$ is an identity mapping, then the above definitions reduce to those of a self-mapping $T$. By using the following iterative algorithm:

$$
x_{n+1}=P\left(\left(1-\alpha_{n}\right) x_{n}+\alpha_{n} T(P T)^{n-1} x_{n}\right), x_{1} \in K, n \geq 1,
$$

Chidume et al. [15] gave some strong and weak convergence theorems for nonself asymptotically nonexpansive mappings in uniformly convex Banach spaces. They also established a demiclosedness principle.

Recently, Temir $[4,5]$ introduced the following definitions:

Definition 1.2. Let $K$ be a nonempty subset of real normed linear space $E$. For self mappings $T, I: K \rightarrow K$,

(1) $T$ is called be asymptotically $I$-nonexpansive $[4,5]$ on $K$ if there exists a sequence $\left\{v_{n}\right\} \subset[0, \infty)$ with $\lim _{n \rightarrow \infty} v_{n}=0$ such that

$$
\left\|T^{n} x-T^{n} y\right\| \leq\left(1+v_{n}\right)\left\|I^{n} x-I^{n} y\right\|
$$

for all $x, y \in K$ and $n \geq 1$.

(2) $T$ is called $I$-uniformly Lipschitz if there exists $\Gamma>0$ such that

$$
\left\|T^{n} x-T^{n} y\right\| \leq \Gamma\left\|I^{n} x-I^{n} y\right\|, x, y \in K \text { and } n \geq 1 .
$$


In 2007, Agarwal et al.[2] introduced the following iteration scheme:

$$
\left\{\begin{array}{l}
x_{1}=x \in K \\
x_{n+1}=\left(1-\alpha_{n}\right) T^{n} x_{n}+\alpha_{n} T^{n} y_{n}, \\
y_{n}=\left(1-\beta_{n}\right) x_{n}+\beta_{n} T^{n} x_{n}, \quad n \geq 1 .
\end{array}\right.
$$

where $\left\{\alpha_{n}\right\}$ and $\left\{\beta_{n}\right\}$ are in $(0,1)$. They showed that this scheme converges at a rate same as that of Picard iteration. Also, Agarwal et al.[2] showed that this scheme converges faster than Mann and Ishikawa iteration for contractions. Khan and Abbas [3] generalized corresponding theorems of Agarwal et al. [2] to the case of two mappings.

Recently, Temir [4] studied the weak and strong convergence of implicit iteration process to a common fixed point for a finite family of asymptotically $I$-nonexpansive mappings in Banach spaces. In [7], Gu proved some convergence theorems of non-implicit iteration process with errors for a finite families of $I$-asymptotically nonexpansive mappings in Banach spaces. In [6], Yang and Xie first introduced the class of $I$-asymptotically nonexpansive nonself-maps, then proved convergence theorems of an iteration process to a common fixed points of a finite family of $I$-asymptotically nonexpansive nonself-mappings.

Definition 1.3 ([6]). Let $K$ be a nonempty subset of a real normed space $X$ and $P: X \rightarrow K$ be a nonexpansive retraction of $X$ onto $K$. Let $T, I$ : $K \rightarrow X$ be two mappings.

(1) $T$ is called nonself $I$-asymptotically nonexpansive if there exists sequence $\left\{u_{n}\right\} \subset[0, \infty)$ with $\lim _{n \rightarrow \infty} u_{n}=0$, such that

$$
\left\|T(P T)^{n-1} x-T(P T)^{n-1} y\right\| \leq\left(1+u_{n}\right)\left\|I(P I)^{n-1} x-I(P I)^{n-1} y\right\|
$$

for all $x, y \in K$ and $n \geq 1$.

(2) $T$ is said to be uniformly $\Gamma$-Lipschitzian if there exists $\Gamma>0$ such that for all $x, y \in K$ and all positive integer $n$

$$
\left\|T(P T)^{n-1} x-T(P T)^{n-1} y\right\| \leq \Gamma\left\|I(P I)^{n-1} x-I(P I)^{n-1} y\right\| .
$$

Motivated and inspired by the above works, in this paper, we introduce a new explicit iterative sequence $\left\{x_{n}\right\}$ as follows:

Let $K$ be a nonempty subset of a Banach space $X$. Let $\left\{T_{i}\right\}_{i}^{N}: K \rightarrow X$ be $N$ nonself $I_{i}$-asymptotically nonexpansive mappings and $\left\{I_{i}\right\}_{i=1}^{N}: K \rightarrow X$ be $N$ nonself asymptotically nonexpansive mappings. Let $\left\{\alpha_{n}\right\}$ and $\left\{\beta_{n}\right\}$ be two real sequences in $[0,1]$. Then the sequence $\left\{x_{n}\right\}$ is generated as follows:

$$
\left\{\begin{array}{l}
x_{n+1}=P\left(\left(1-\alpha_{n}\right) T_{i}\left(P T_{i}\right)^{n-1} x_{n}+\alpha_{n} I_{i}\left(P I_{i}\right)^{n-1} y_{n}\right) \\
y_{n}=P\left(\left(1-\beta_{n}\right) x_{n}+\beta_{n} T_{i}\left(P T_{i}\right)^{n-1} x_{n}\right)
\end{array}, \quad n \geq 1,\right.
$$


where $n=(k-1) N+i, i=i(n) \in J:=\{1,2, \ldots, N\}$ is a positive integer and $k(n) \rightarrow \infty$ as $n \rightarrow \infty$. Thus, (1) can be expressed in the following form:

$$
\left\{\begin{array}{l}
x_{n+1}=P\left(\left(1-\alpha_{n}\right) T_{i}\left(P T_{i}\right)^{k(n)-1} x_{n}+\alpha_{n} I_{i}\left(P I_{i}\right)^{k(n)-1} y_{n}\right) \\
y_{n}=P\left(\left(1-\beta_{n}\right) x_{n}+\beta_{n} T_{i}\left(P T_{i}\right)^{k(n)-1} x_{n}\right)
\end{array}, \quad n \geq 1 .\right.
$$

In this paper, we get some weak and strong convergence theorems of this iterative sequence for a family of nonself asymptotically $I$-nonexpansive mappings $\left\{T_{i}\right\}_{i}^{N}$ and a family of nonself asymptotically nonexpansive mappings $\left\{I_{i}\right\}_{i}^{N}$. Our theorems also are new in case of self-mappings.

\section{Preliminaries AND NOtations}

In order to prove the main results of this work, we need the following some concepts and results:

A Banach space $X$ is said to satisfy Opial's condition if, for any sequence $\left\{x_{n}\right\}$ in $X, x_{n} \rightarrow x$ implies that

$$
\limsup _{n \rightarrow \infty}\left\|x_{n}-x\right\|<\limsup _{n \rightarrow \infty}\left\|x_{n}-y\right\|
$$

for all $y \in X$ with $y \neq x$, where $x_{n} \rightarrow x$ means that $\left\{x_{n}\right\}$ converges weakly to $x$.

A Banach space $X$ is said to have a Fréchet differentiable norm [10] if for all $x \in S_{X}=\{x \in X:\|x\|=1\}$

$$
\lim _{t \rightarrow 0} \frac{\|x+t y\|-\|x\|}{t}
$$

exists and is attained uniformly in $y \in S_{X}$.

A mapping $T$ with domain $D(T)$ and range $R(T)$ in $X$ is said to be demiclosed at $p$ if whenever $\left\{x_{n}\right\}$ is a sequence in $D(T)$ such that $x_{n} \rightarrow$ $x^{*} \in D(T)$ and $T x_{n} \rightarrow p$ then $T x^{*}=p$.

A mapping $T: K \rightarrow K$ is said to be semicompact if, for any bounded sequence $\left\{x_{n}\right\}$ in $K$ such that $\left\|x_{n}-T x_{n}\right\| \rightarrow 0$ as $n \rightarrow \infty$, there exists a subsequence say $\left\{x_{n_{j}}\right\}$ of $\left\{x_{n}\right\}$ such that $\left\{x_{n_{j}}\right\}$ converges strongly to some $x^{*}$ in $K$.

The mapping $T: K \rightarrow K$ with $F(T) \neq \emptyset$ is said to satisfy condition $(A)$ [11] if there is a nondecreasing function $f:[0, \infty) \rightarrow[0, \infty)$ with $f(0)=0$, $f(t)>0$ for all $t \in(0, \infty)$ such that $\|x-T x\| \geq f(d(x, F(T)))$ for all $n \geq 1$. Senter and Dotson [11] pointed out that every continuous and semicompact mapping must satisfy Condition (A).

Two mappings $T, S: K \rightarrow K$ are said to satisfy condition $\left(A^{\prime}\right)[12]$ if there is a nondecreasing function $f:[0, \infty) \rightarrow[0, \infty)$ with $f(0)=0, f(t)>0$ for all $t \in(0, \infty)$ such that

$$
\frac{1}{2}\left(\left\|x-T_{1} x\right\|+\left\|x-T_{2} x\right\|\right) \geq f(d(x, F))
$$


for all $x \in K$, where $d(x, F)=\inf \{\|x-p\|: p \in F:=F(T) \cap F(S)\}$.

We modify these definitions for our case as follows:

A family $\left\{T_{i}\right\}_{i}^{N}: K \rightarrow X$ be $N$ nonself $I_{i}$-asymptotically nonexpansive mappings and a family $\left\{I_{i}\right\}_{i}^{N}: K \rightarrow X$ be $N$ nonself asymptotically nonexpansive mappings with $F=\bigcap_{i=1}^{N} F\left(T_{i}\right) \cap F\left(I_{i}\right) \neq \emptyset$ are said to satisfy condition (B) if there is a nondecreasing function $f:[0, \infty) \rightarrow[0, \infty)$ with $f(0)=0, f(t)>0$ for all $t \in(0, \infty)$ such that

$$
\max _{1 \leq i \leq N}\left\|\frac{1}{2}\left(\left\|x-T_{i} x\right\|+\left\|x-I_{i} x\right\|\right)\right\| \geq f(d(x, F)) .
$$

Lemma 2.1 ([8]). Let $\left\{a_{n}\right\},\left\{b_{n}\right\}$ and $\left\{\delta_{n}\right\}$ be sequences of nonnegative real numbers satisfying the inequality

$$
a_{n+1} \leq\left(1+\delta_{n}\right) a_{n}+b_{n}, \quad n \geq 1 .
$$

If $\sum_{n=1}^{\infty} b_{n}<\infty$ and $\sum_{n=1}^{\infty} \delta_{n}<\infty$, then $\lim _{n \rightarrow \infty} a_{n}$ exists.

Lemma $2.2([9])$. Suppose that $X$ is a uniformly convex Banach space and $0<p \leq t_{n} \leq q<1$ for all $n \geq 1$. Also, suppose that $\left\{x_{n}\right\}$ and $\left\{y_{n}\right\}$ are sequences of $X$ such that

$$
\limsup _{n \rightarrow \infty}\left\|x_{n}\right\| \leq r, \limsup _{n \rightarrow \infty}\left\|y_{n}\right\| \leq r \text { and } \lim _{n \rightarrow \infty}\left\|t_{n} x_{n}+\left(1-t_{n}\right) y_{n}\right\|=r
$$

hold for some $r \geq 0$. Then $\lim _{n \rightarrow \infty}\left\|x_{n}-y_{n}\right\|=0$.

Lemma 2.3 ([13]). Let $X$ be a uniformly convex Banach space and $K a$ convex subset of $X$. Then there exists a strictly increasing continuous convex function $\phi:[0, \infty) \rightarrow[0, \infty)$ with $\phi(0)=0$ such that for each $S: K \rightarrow K$ with Lipschitz constant $L$,

$$
\|\alpha S x+(1-\alpha) S y-S[\alpha x+(1-\alpha) y]\| \leq L \phi^{-1}\left(\|x-y\|+\frac{1}{L}\|S x-S y\|\right)
$$

for all $x, y \in K$ and $0<\alpha<1$.

Lemma 2.4 ([15]). Let $X$ be a real uniformly convex Banach space, $K$ a nonempty closed subset of $E$, and let $T: K \rightarrow X$ be an asymptotically nonexpansive mapping with a sequence $\left\{k_{n}\right\} \subset[1, \infty)$ and $k_{n} \rightarrow 1$ as $n \rightarrow \infty$, then $(E-T)$ is demiclosed at zero, where $E$ is an identity mapping.

A Banach space $X$ is said to have the Kadec-Klee property if, for every sequence $\left\{x_{n}\right\}$ in $X, x_{n} \rightarrow x$ and $\left\|x_{n}\right\| \rightarrow\|x\|$ imply $\left\|x_{n}-x\right\| \rightarrow 0$. Every locally uniformly convex space has the Kadec-Klee property. In particular, $L_{p}$ spaces, $1<p<\infty$ have this property.

Lemma 2.5 ([13]). Let $X$ be a real reflexive Banach space such that its dual $X$ has Kadec-Klee property. Let $\left\{x_{n}\right\}$ be a bounded sequence in $X$ and $q_{1}, q_{2} \in \omega_{w}\left\{x_{n}\right\}$ (weak limit set of $\left\{x_{n}\right\}$ ). Suppose $\lim _{n \rightarrow \infty}\left\|\alpha x_{n}+(1-\alpha) q_{1}-q_{2}\right\|$ exists for all $\alpha \in[0,1]$. Then $q_{1}=q_{2}$. 


\section{Strong CONVERGEnCE TheOREMS}

First, we prove the following lemmas.

Lemma 3.1. Let $X$ be a real Banach space, $K$ be a nonempty closed convex subset of $X$ which is also a nonexpansive retract with retraction $P$. Let $\left\{T_{i}\right\}_{i}^{N}: K \rightarrow X$ be $N$ nonself $I_{i}$-asymptotically nonexpansive mappings with sequences $\left\{l_{n}^{(i)}\right\} \subset[0, \infty)$ such that $\sum_{n=1}^{\infty} l_{n}^{(i)}<\infty$ and $\left\{I_{i}\right\}_{i}^{N}: K \rightarrow X$ be $N$ nonself asymptotically nonexpansive mappings with sequences $\left\{k_{n}^{(i)}\right\} \subset$ $[0, \infty)$ such that $\sum_{n=1}^{\infty} k_{n}^{(i)}<\infty$. Suppose that for any given $x_{1} \in K$, the sequence $\left\{x_{n}\right\}$ is generated by $(1)$ and $F=\bigcap_{i=1}^{N} F\left(T_{i}\right) \cap F\left(I_{i}\right) \neq \emptyset$. Then

(i) $\lim _{n \rightarrow \infty}\left\|x_{n}-q\right\|$ exists for all $q \in F$.

(ii) $\lim _{n \rightarrow \infty} d\left(x_{n}, F\right)$ exists, where $d\left(x_{n}, F\right)=\inf _{p \in F}\left\|x_{n}-p\right\|$.

Proof. (i) Let $q \in F$. Setting

$$
l_{n}=\max \left\{l_{n}^{(1)}, l_{n}^{(2)}, \ldots, l_{n}^{(N)}\right\}, k_{n}=\max \left\{k_{n}^{(1)}, k_{n}^{(2)}, \ldots, k_{n}^{(N)}\right\} .
$$

Since $\sum_{n=1}^{\infty} l_{n}^{(i)}<\infty, \sum_{n=1}^{\infty} k_{n}^{(i)}<\infty$, so $\sum_{n=1}^{\infty} l_{n}<\infty, \sum_{n=1}^{\infty} k_{n}<\infty$. Using (1), we have

$$
\begin{aligned}
\left\|y_{n}-q\right\| & =\left\|P\left(\left(1-\beta_{n}\right) x_{n}+\beta_{n} T_{i}\left(P T_{i}\right)^{n-1} x_{n}\right)-P q\right\| \\
& \leq\left\|\left(1-\beta_{n}\right) x_{n}+\beta_{n} T_{i}\left(P T_{i}\right)^{n-1} x_{n}-q\right\| \\
& \leq\left(1-\beta_{n}\right)\left\|x_{n}-q\right\|+\beta_{n}\left\|T_{i}\left(P T_{i}\right)^{n-1} x_{n}-q\right\| \\
& \leq\left(1-\beta_{n}\right)\left\|x_{n}-q\right\|+\beta_{n}\left(1+l_{n}\right)\left\|I_{i}\left(P I_{i}\right)^{n-1} x_{n}-q\right\| \\
& \leq\left(1-\beta_{n}\right)\left\|x_{n}-q\right\|+\beta_{n}\left(1+l_{n}\right)\left(1+k_{n}\right)\left\|x_{n}-q\right\| \\
& =\left(1+\beta_{n} l_{n}+\beta_{n} k_{n}+\beta_{n} l_{n} k_{n}\right)\left\|x_{n}-q\right\| \\
& \leq\left(1+l_{n}+k_{n}+l_{n} k_{n}\right)\left\|x_{n}-q\right\| \\
& =\left(1+l_{n}\right)\left(1+k_{n}\right)\left\|x_{n}-q\right\|,
\end{aligned}
$$

and so

$$
\begin{aligned}
\left\|x_{n+1}-q\right\| & =\left\|P\left(\left(1-\alpha_{n}\right) T_{i}\left(P T_{i}\right)^{n-1} x_{n}+\alpha_{n} I_{i}\left(P I_{i}\right)^{n-1} y_{n}\right)-P q\right\| \\
& \leq\left\|\left(1-\alpha_{n}\right) T_{i}\left(P T_{i}\right)^{n-1} x_{n}+\alpha_{n} I_{i}\left(P I_{i}\right)^{n-1} y_{n}-q\right\| \\
& \leq\left(1-\alpha_{n}\right)\left\|T_{i}\left(P T_{i}\right)^{n-1} x_{n}-q\right\|+\alpha_{n}\left\|I_{i}\left(P I_{i}\right)^{n-1} y_{n}-q\right\| \\
& \leq\left(1-\alpha_{n}\right)\left(1+l_{n}\right)\left\|I_{i}\left(P I_{i}\right)^{n-1} x_{n}-q\right\|
\end{aligned}
$$




$$
\begin{aligned}
& +\alpha_{n}\left(1+k_{n}\right)\left\|y_{n}-q\right\| \\
\leq & \left(1-\alpha_{n}\right)\left(1+l_{n}\right)\left(1+k_{n}\right)\left\|x_{n}-q\right\| \\
& \quad+\alpha_{n}\left(1+k_{n}\right)\left(1+l_{n}\right)\left(1+k_{n}\right)\left\|x_{n}-q\right\| \\
= & \left(1+k_{n}\right)\left(1+l_{n}\right)\left[\left(1-\alpha_{n}\right)\left\|x_{n}-q\right\|+\alpha_{n}\left(1+k_{n}\right)\left\|x_{n}-q\right\|\right] \\
\leq & \left(1+k_{n}\right)\left(1+l_{n}\right)\left(1+\alpha_{n} k_{n}\right)\left\|x_{n}-q\right\| \\
\leq & \left(1+l_{n}+2 k_{n}+2 l_{n} k_{n}+k_{n}^{2}+l_{n} k_{n}^{2}\right)\left\|x_{n}-q\right\| \\
= & \left(1+\delta_{n}\right)\left\|x_{n}-q\right\|,
\end{aligned}
$$

where $\delta_{n}=l_{n}+2 k_{n}+2 l_{n} k_{n}+k_{n}^{2}+l_{n} k_{n}^{2}$.

Since $\sum_{n=1}^{\infty} l_{n}<\infty$ and $\sum_{n=1}^{\infty} k_{n}<\infty$, we obtain $\sum_{n=1}^{\infty} \delta_{n}<\infty$. By Lemma 2.1 and (3), we get $\lim _{n \rightarrow \infty}\left\|x_{n}-q\right\|$ exists.

(ii) Taking infimum over all $q \in F$ in (3), we have

$$
d\left(x_{n+1}, F\right) \leq\left(1+\delta_{n}\right) d\left(x_{n}, F\right) .
$$

It follows from (4) and Lemma 2.1 that $\lim _{n \rightarrow \infty} d\left(x_{n}, F\right)$ exists.

Lemma 3.2. Let $X$ be a real uniformly convex Banach space, $K$ be a nonempty closed convex subset of $X$ which is also a nonexpansive retract with retraction $P$. Let $\left\{T_{i}\right\}_{i}^{N}: K \rightarrow X$ be $N$ nonself $I_{i}$-asymptotically nonexpansive mappings with sequences $\left\{l_{n}^{(i)}\right\} \subset[0, \infty)$ such that $\sum_{n=1}^{\infty} l_{n}^{(i)}<\infty$ and $\left\{I_{i}\right\}_{i}^{N}: K \rightarrow X$ be $N$ nonself asymptotically nonexpansive mappings with sequences $\left\{k_{n}^{(i)}\right\} \subset[0, \infty)$ such that $\sum_{n=1}^{\infty} k_{n}^{(i)}<\infty$. Let $\left\{\alpha_{n}\right\}$ and $\left\{\beta_{n}\right\}$ be sequences in $[a, 1-a]$ for some $a \in(0,1)$. Suppose that for any given $x_{1} \in K$, the sequence $\left\{x_{n}\right\}$ is generated by $(1)$ and $F=\bigcap_{i=1}^{N} F\left(T_{i}\right) \cap F\left(I_{i}\right) \neq \emptyset$. Then,

$$
\lim _{n \rightarrow \infty}\left\|x_{n}-T_{i} x_{n}\right\|=\lim _{n \rightarrow \infty}\left\|x_{n}-I_{i} x_{n}\right\|=0 \text { for all } i \in J .
$$

Proof. By Lemma 3.1, $\lim _{n \rightarrow \infty}\left\|x_{n}-q\right\|$ exists for all $q \in F$. Call it $c$. Taking lim sup on both sides in the inequality (2), we get

$$
\lim \sup _{n \rightarrow \infty}\left\|y_{n}-q\right\| \leq \lim \sup _{n \rightarrow \infty}\left\|x_{n}-q\right\|=\lim _{n \rightarrow \infty}\left\|x_{n}-q\right\|=c .
$$

Thus

$$
\begin{aligned}
\left\|T_{i}\left(P T_{i}\right)^{n-1} x_{n}-q\right\| & \leq\left(1+l_{n}\right)\left\|I_{i}\left(P I_{i}\right)^{n-1} x_{n}-q\right\| \\
& \leq\left(1+l_{n}\right)\left(1+k_{n}\right)\left\|x_{n}-q\right\|
\end{aligned}
$$

for all $n \geq 1$ implies that

$$
\lim \sup _{n \rightarrow \infty}\left\|T_{i}\left(P T_{i}\right)^{n-1} x_{n}-q\right\| \leq c .
$$


Next, $\left\|I_{i}\left(P I_{i}\right)^{n-1} y_{n}-q\right\| \leq\left(1+k_{n}\right)\left\|y_{n}-q\right\|$ gives by (5) that

$$
\lim \sup _{n \rightarrow \infty}\left\|I_{i}\left(P I_{i}\right)^{n-1} y_{n}-q\right\| \leq c .
$$

From (3), we have

$$
\begin{aligned}
\left\|x_{n+1}-q\right\| & \leq\left\|\left(1-\alpha_{n}\right)\left(T_{i}\left(P T_{i}\right)^{n-1} x_{n}-q\right)+\alpha_{n}\left(I_{i}\left(P I_{i}\right)^{n-1} y_{n}-q\right)\right\| \\
& \leq\left(1+\delta_{n}\right)\left\|x_{n}-q\right\| .
\end{aligned}
$$

Since $\sum_{n=1}^{\infty} \delta_{n}<\infty$ and $\lim _{n \rightarrow \infty}\left\|x_{n+1}-q\right\|=c$, letting $n \rightarrow \infty$ in the inequality (8), we have

(9) $\lim _{n \rightarrow \infty}\left\|\left(1-\alpha_{n}\right)\left(T_{i}\left(P T_{i}\right)^{n-1} x_{n}-q\right)+\alpha_{n}\left(I_{i}\left(P I_{i}\right)^{n-1} y_{n}-q\right)\right\|=c$.

By using (6), (7), (9) and Lemma 2.2, we get

$$
\lim _{n \rightarrow \infty}\left\|T_{i}\left(P T_{i}\right)^{n-1} x_{n}-I_{i}\left(P I_{i}\right)^{n-1} y_{n}\right\|=0 .
$$

Using (1), we have

$$
\begin{aligned}
\left\|x_{n+1}-q\right\| & \leq\left\|\left(1-\alpha_{n}\right) T_{i}\left(P T_{i}\right)^{n-1} x_{n}+\alpha_{n} I_{i}\left(P I_{i}\right)^{n-1} y_{n}-q\right\| \\
\text { (11) } & \leq\left\|T_{i}\left(P T_{i}\right)^{n-1} x_{n}-q\right\|+\alpha_{n}\left\|T_{i}\left(P T_{i}\right)^{n-1} x_{n}-I_{i}\left(P I_{i}\right)^{n-1} y_{n}\right\|
\end{aligned}
$$

Taking the liminf on both sides in (11), by (10) and $\lim _{n \rightarrow \infty}\left\|x_{n+1}-q\right\|=c$, we obtain

$$
c \leq \lim \inf _{n \rightarrow \infty}\left\|T_{i}\left(P T_{i}\right)^{n-1} x_{n}-q\right\| .
$$

This together with (6) implies that

$$
\lim _{n \rightarrow \infty}\left\|T_{i}\left(P T_{i}\right)^{n-1} x_{n}-q\right\|=c .
$$

Further,

$$
\begin{aligned}
\left\|T_{i}\left(P T_{i}\right)^{n-1} x_{n}-q\right\| \leq & \left\|T_{i}\left(P T_{i}\right)^{n-1} x_{n}-I_{i}\left(P I_{i}\right)^{n-1} y_{n}\right\| \\
& +\left\|I_{i}\left(P I_{i}\right)^{n-1} y_{n}-q\right\| \\
\leq & \left\|T_{i}\left(P T_{i}\right)^{n-1} x_{n}-I_{i}\left(P I_{i}\right)^{n-1} y_{n}\right\| \\
& +\left(1+k_{n}\right)\left\|y_{n}-q\right\|
\end{aligned}
$$

gives that

$$
c \leq \lim \inf _{n \rightarrow \infty}\left\|y_{n}-q\right\| .
$$

By (5) and (14), we obtain

$$
\lim _{n \rightarrow \infty}\left\|y_{n}-q\right\|=c .
$$


From (3), we have

$$
\begin{aligned}
\left\|y_{n}-q\right\| & \leq\left\|\left(1-\beta_{n}\right)\left(x_{n}-q\right)+\beta_{n}\left(T_{i}\left(P T_{i}\right)^{n-1} x_{n}-q\right)\right\| \\
& \leq\left(1+l_{n}\right)\left(1+k_{n}\right)\left\|x_{n}-q\right\| .
\end{aligned}
$$

Thus from (15), we get

$$
\lim _{n \rightarrow \infty}\left\|\left(1-\beta_{n}\right)\left(x_{n}-q\right)+\beta_{n}\left(T_{i}\left(P T_{i}\right)^{n-1} x_{n}-q\right)\right\|=c
$$

Hence, using $\lim _{n \rightarrow \infty}\left\|x_{n}-q\right\|=c,(13)$, (16) and Lemma 2.2, we obtain

$$
\lim _{n \rightarrow \infty}\left\|x_{n}-T_{i}\left(P T_{i}\right)^{n-1} x_{n}\right\|=0 \text {. }
$$

By $y_{n}=P\left(\left(1-\beta_{n}\right) x_{n}+\beta_{n} T_{i}\left(P T_{i}\right)^{n-1} x_{n}\right)$ and $(17)$, since $P$ is nonexpansive mapping, we have

$$
\begin{aligned}
\left\|y_{n}-x_{n}\right\| & =\left\|P\left(\left(1-\beta_{n}\right) x_{n}+\beta_{n} T_{i}\left(P T_{i}\right)^{n-1} x_{n}\right)-P x_{n}\right\| \\
& \leq\left\|\left(1-\beta_{n}\right) x_{n}+\beta_{n} T_{i}\left(P T_{i}\right)^{n-1} x_{n}-x_{n}\right\| \\
& \leq \beta_{n}\left\|T_{i}\left(P T_{i}\right)^{n-1} x_{n}-x_{n}\right\| \\
& \rightarrow 0(n \rightarrow \infty) .
\end{aligned}
$$

Using a similar method, together with (10) and (17), we have

$$
\begin{aligned}
\left\|x_{n+1}-x_{n}\right\| \leq & \left\|P\left(\left(1-\alpha_{n}\right) T_{i}\left(P T_{i}\right)^{n-1} x_{n}+\alpha_{n} I_{i}\left(P I_{i}\right)^{n-1} y_{n}\right)-P x_{n}\right\| \\
\leq & \left\|\left(1-\alpha_{n}\right) T_{i}\left(P T_{i}\right)^{n-1} x_{n}+\alpha_{n} I_{i}\left(P I_{i}\right)^{n-1} y_{n}-x_{n}\right\| \\
\leq & \left\|T_{i}\left(P T_{i}\right)^{n-1} x_{n}-x_{n}\right\| \\
& \quad+\alpha_{n}\left\|T_{i}\left(P T_{i}\right)^{n-1} x_{n}-I_{i}\left(P I_{i}\right)^{n-1} y_{n}\right\| \\
& \rightarrow 0 \text { as } n \rightarrow \infty .
\end{aligned}
$$

It follows from (18) and (19) that

$$
\lim _{n \rightarrow \infty}\left\|x_{n+1}-y_{n}\right\|=0 \text {. }
$$

Furthermore, from (19) and

$$
\begin{aligned}
\left\|x_{n}-I_{i}\left(P I_{i}\right)^{n-1} y_{n}\right\| \leq & \left\|x_{n}-T_{i}\left(P T_{i}\right)^{n-1} x_{n}\right\| \\
& +\left\|T_{i}\left(P T_{i}\right)^{n-1} x_{n}-I_{i}\left(P I_{i}\right)^{n-1} y_{n}\right\| \rightarrow 0
\end{aligned}
$$

we obtain

(21) $\left\|x_{n+1}-I_{i}\left(P I_{i}\right)^{n-1} y_{n}\right\| \leq\left\|x_{n+1}-x_{n}\right\|+\left\|x_{n}-I_{i}\left(P I_{i}\right)^{n-1} y_{n}\right\| \rightarrow 0$. 
Notice that

$$
\begin{aligned}
&\left\|x_{n}-I_{i}\left(P I_{i}\right)^{n-1} x_{n}\right\| \leq\left\|x_{n}-x_{n+1}\right\|+\left\|x_{n+1}-I_{i}\left(P I_{i}\right)^{n-1} y_{n}\right\| \\
&+\left\|I_{i}\left(P I_{i}\right)^{n-1} y_{n}-I_{i}\left(P I_{i}\right)^{n-1} x_{n}\right\| \\
& \leq\left\|x_{n}-x_{n+1}\right\|+\left\|x_{n+1}-I_{i}\left(P I_{i}\right)^{n-1} y_{n}\right\| \\
& \text { qquad }+\left(1+k_{n}\right)\left\|y_{n}-x_{n}\right\| .
\end{aligned}
$$

It follows from (18), (19) and (21) that

$$
\lim _{n \rightarrow \infty}\left\|x_{n}-I_{i}\left(P I_{i}\right)^{n-1} x_{n}\right\|=0 .
$$

Since an asymptotically nonexpansive mapping with respect to $P$ must be uniformly Lipschitzian with respect to $P$, then we have

$$
\begin{aligned}
\left\|x_{n+1}-I_{i} x_{n+1}\right\| \leq & \left\|x_{n+1}-I_{i}\left(P I_{i}\right)^{n} x_{n+1}\right\|+\left\|I_{i}\left(P I_{i}\right)^{n} x_{n+1}-I_{i} x_{n+1}\right\| \\
\leq & \left\|x_{n+1}-I_{i}\left(P I_{i}\right)^{n} x_{n+1}\right\|+L\left\|I_{i}\left(P I_{i}\right)^{n-1} x_{n+1}-x_{n+1}\right\| \\
\leq & \left\|x_{n+1}-I_{i}\left(P I_{i}\right)^{n} x_{n+1}\right\| \\
& \quad+L\left(\left\|I_{i}\left(P I_{i}\right)^{n-1} x_{n+1}-I_{i}\left(P I_{i}\right)^{n-1} y_{n}\right\|\right. \\
& \left.\quad+\left\|I_{i}\left(P I_{i}\right)^{n-1} y_{n}-x_{n+1}\right\|\right) \\
\leq & \left\|x_{n+1}-I_{i}\left(P I_{i}\right)^{n} x_{n+1}\right\|+L^{2}\left\|x_{n+1}-y_{n}\right\| \\
& \quad+L\left\|I_{i}\left(P I_{i}\right)^{n-1} y_{n}-x_{n+1}\right\| .
\end{aligned}
$$

Taking $\lim _{n \rightarrow \infty}$ on both sides in the above inequality, then from (20), (21) and (22) we get

$$
\lim _{n \rightarrow \infty}\left\|x_{n}-I_{i} x_{n}\right\|=0 .
$$

Now we make use of the fact that every nonself $I$-asymptotically nonexpansive mapping with respect to $P$ must be $I$-uniformly Lipschitz with respect to $P$. Then

$$
\begin{aligned}
&\left\|x_{n+1}-T_{i} x_{n+1}\right\| \leq\left\|x_{n+1}-T_{i}\left(P T_{i}\right)^{n} x_{n+1}\right\| \\
&+\left\|T_{i}\left(P T_{i}\right)^{n} x_{n+1}-T_{i}\left(P T_{i}\right)^{n} x_{n}\right\| \\
& \quad\left\|T_{i}\left(P T_{i}\right)^{n} x_{n}-T_{i} x_{n+1}\right\| \\
& \leq \| x_{n+1}-T_{i}\left(P T_{i}\right)^{n} x_{n+1} \| \\
& \quad+\Gamma\left\|I_{i}\left(P I_{i}\right)^{n} x_{n+1}-I_{i}\left(P I_{i}\right)^{n} x_{n}\right\| \\
& \quad+\Gamma\left\|I_{i}\left(P I_{i}\right)^{n} x_{n}-I_{i} x_{n+1}\right\| \\
& \leq\left\|x_{n+1}-T_{i}\left(P T_{i}\right)^{n} x_{n+1}\right\|+\Gamma L\left\|x_{n+1}-x_{n}\right\| \\
& \quad+\Gamma L\left\|I_{i}\left(P I_{i}\right)^{n-1} x_{n}-x_{n+1}\right\| \\
& \leq\left\|x_{n+1}-T_{i}\left(P T_{i}\right)^{n} x_{n+1}\right\|+\Gamma L\left\|x_{n+1}-x_{n}\right\|
\end{aligned}
$$




$$
+\Gamma L\left(\left\|I_{i}\left(P I_{i}\right)^{n-1} x_{n}-x_{n}\right\|+\left\|x_{n}-x_{n+1}\right\|\right)
$$

yields by (17), (19) and (22)

$$
\lim _{n \rightarrow \infty}\left\|x_{n}-T_{i} x_{n}\right\|=0 .
$$

We now prove strong convergence theorems of the iterative scheme (1) in Banach space and uniformly convex Banach spaces.

Theorem 3.1. Let $X$ be a real Banach space, $K$ be a nonempty closed convex subset of $X$ which is also a nonexpansive retract with retraction $P$. Let $\left\{T_{i}\right\}_{i}^{N}: K \rightarrow X$ be $N$ nonself $I_{i}$-asymptotically nonexpansive mappings with sequences $\left\{l_{n}^{(i)}\right\} \subset[0, \infty)$ such that $\sum_{n=1}^{\infty} l_{n}^{(i)}<\infty$ and $\left\{I_{i}\right\}_{i}^{N}: K \rightarrow X$ be $N$ nonself asymptotically nonexpansive mappings with sequences $\left\{k_{n}^{(i)}\right\} \subset$ $[0, \infty)$ such that $\sum_{n=1}^{\infty} k_{n}^{(i)}<\infty$. Suppose that for any given $x_{1} \in K$, the sequence $\left\{x_{n}\right\}$ is generated by $(1)$ and $F=\bigcap_{i=1}^{N} F\left(T_{i}\right) \cap F\left(I_{i}\right) \neq \emptyset$. Then, $\left\{x_{n}\right\}$ converges strongly to a common fixed point of $\left\{T_{i}\right\}_{i}^{N}$ and $\left\{I_{i}\right\}_{i}^{N}$ if and only if $\liminf _{n \rightarrow \infty} d\left(x_{n}, F\right)=0$.

Proof. The necessity of Theorem is obvious. Let us proof the sufficiency part of theorem. Let $q \in F$. Then by Lemma 2.1 (ii), $\lim _{n \rightarrow \infty} d\left(x_{n}, F\right)$ exists and by assumption $\liminf _{n \rightarrow \infty} d\left(x_{n}, F\right)=0$, we obtain $\lim _{n \rightarrow \infty} d\left(x_{n}, F\right)=0$.

Next, we show that $\left\{x_{n}\right\}$ is a Cauchy sequence in $K$. Notice that from (3) for any $q \in K$, we have

$$
\begin{aligned}
\left\|x_{n+m}-q\right\| & \leq \exp \left(\sum_{n=1}^{\infty} \delta_{n}\right)\left\|x_{n}-q\right\| \\
& <M\left\|x_{n}-q\right\|
\end{aligned}
$$

for all $m, n$, where $M=\exp \left(\sum_{n=1}^{\infty} \delta_{n}\right)+1<\infty$. Since $\lim _{n \rightarrow \infty} d\left(x_{n}, F\right)=0$, for any given $\varepsilon>0$, there exists a positive integer $N_{0}$ such that for all $n \geq N_{0}, d\left(x_{n}, F\right)<\frac{\varepsilon}{2 M}$. There exists $q_{1} \in F$ such that $\left\|x_{n_{0}}-q_{1}\right\|<\frac{\varepsilon}{2 M}$.

Hence, for all $n \geq N_{0}$ and $m \geq 1$, we have

$$
\begin{aligned}
\left\|x_{n+m}-x_{n}\right\| & \leq\left\|x_{n+m}-q_{1}\right\|+\left\|x_{n}-q_{1}\right\| \\
& \leq M\left\|x_{n_{0}}-q_{1}\right\|+M\left\|x_{n_{0}}-q_{1}\right\| \\
& \leq 2 M\left\|x_{n_{0}}-q_{1}\right\| \\
& \leq 2 M \frac{\varepsilon}{2 M}=\varepsilon
\end{aligned}
$$


which implies that $\left\{x_{n}\right\}$ is a Cauchy sequence in $K$.

Thus, the completeness of $X$ implies that $\left\{x_{n}\right\}$ is convergent. Assume that $\left\{x_{n}\right\}$ converges to a point $q$. Then $q \in K$, because $K$ is closed subset of $X$. And $\lim _{n \rightarrow \infty} d\left(x_{n}, F\right)=0$ implies that $\lim _{n \rightarrow \infty} d(q, F)=0$. $\mathrm{F}$ is closed, thus $q \in F$. This completes the proof.

Applying Theorem 3.1, we obtain a strong convergence theorem of the iterative scheme (1) under the condition (B) as follows.

Theorem 3.2. Let $X$ be a real uniformly convex Banach space, $K$ be a nonempty closed convex subset of $X$ which is also a nonexpansive retract with retraction $P$. Let $\left\{T_{i}\right\}_{i}^{N}: K \rightarrow X$ be $N$ nonself $I_{i}$-asymptotically nonexpansive mappings with sequences $\left\{l_{n}^{(i)}\right\} \subset[0, \infty)$ such that $\sum_{n=1}^{\infty} l_{n}^{(i)}<\infty$ and $\left\{I_{i}\right\}_{i}^{N}: K \rightarrow X$ be $N$ nonself asymptotically nonexpansive mappings with sequences $\left\{k_{n}^{(i)}\right\} \subset[0, \infty)$ such that $\sum_{n=1}^{\infty} k_{n}^{(i)}<\infty$. Let $\left\{\alpha_{n}\right\}$ and $\left\{\beta_{n}\right\}$ be sequences in $[a, 1-a]$ for some $a \in(0,1)$. Suppose that for any given $x_{1} \in$ $K$, the sequence $\left\{x_{n}\right\}$ is generated by $(1)$ and $F=\bigcap_{i=1}^{N} F\left(T_{i}\right) \cap F\left(I_{i}\right) \neq \emptyset$. If $\left\{T_{i}\right\}_{i}^{N}$ and $\left\{I_{i}\right\}_{i}^{N}$ satisfy the condition $(B)$, then $\left\{x_{n}\right\}$ converges strongly to a common fixed point of $\left\{T_{i}\right\}_{i}^{N}$ and $\left\{I_{i}\right\}_{i}^{N}$.

Proof. As is proved in Lemma 3.2 that

$$
\lim _{n \rightarrow \infty}\left\|x_{n}-T_{i} x_{n}\right\|=\lim _{n \rightarrow \infty}\left\|x_{n}-I_{i} x_{n}\right\|=0 \text { for all } i \in J .
$$

Because $\left\{T_{i}\right\}_{i}^{N}$ and $\left\{I_{i}\right\}_{i}^{N}$ satisfy Condition (B), therefore

$$
f\left(d\left(x_{n}, F\right)\right) \leq \max _{1 \leq i \leq N}\left\|\frac{1}{2}\left(\left\|x-T_{i} x\right\|+\left\|x-I_{i} x\right\|\right)\right\| .
$$

Thus it follows from $(23)$ that $\lim _{n \rightarrow \infty} f\left(d\left(x_{n}, F\right)\right)=0$.

Since $f:[0, \infty) \rightarrow[0, \infty)$ is a nondecreasing function satisfying $f(0)=0$, $f(r)>0$ for all $r \in(0, \infty)$, therefore we have $\lim _{n \rightarrow \infty} d\left(x_{n}, F\right)=0$.

Now all the conditions of Theorem 3.1 are satisfied, therefore by its conclusion $\left\{x_{n}\right\}$ converges strongly to a point of $F$.

\section{WEAK CONVERGENCE THEOREMS}

Following lemma is the key for our weak convergence result.

Lemma 4.1. Let $X$ be a real uniformly convex Banach space, $K$ be a nonempty closed convex subset of $X$ which is also a nonexpansive retract with retraction $P$. Let $\left\{T_{i}\right\}_{i}^{N}: K \rightarrow X$ be $N$ nonself $I_{i}$-asymptotically nonexpansive mappings with sequences $\left\{l_{n}^{(i)}\right\} \subset[0, \infty)$ such that $\sum_{n=1}^{\infty} l_{n}^{(i)}<\infty$ and $\left\{I_{i}\right\}_{i}^{N}: K \rightarrow X$ be $N$ nonself asymptotically nonexpansive mappings with 
sequences $\left\{k_{n}^{(i)}\right\} \subset[0, \infty)$ such that $\sum_{n=1}^{\infty} k_{n}^{(i)}<\infty$. Let $\left\{\alpha_{n}\right\}$ and $\left\{\beta_{n}\right\}$ be sequences in $[a, 1-a]$ for some $a \in(0,1)$. Suppose that for any given $x_{1} \in K$, the sequence $\left\{x_{n}\right\}$ is generated by $(1)$ and $F=\bigcap_{i=1}^{N} F\left(T_{i}\right) \cap F\left(I_{i}\right) \neq \emptyset$. Then $\lim _{n \rightarrow \infty}\left\|t x_{n}+(1-t) q_{1}-q_{2}\right\|$ exists for all $t \in[0,1]$ and $q_{1}, q_{2} \in F$.

Proof. By Lemma 3.1, $\lim _{n \rightarrow \infty}\left\|x_{n}-q\right\|=c$ for all $q \in F$. Since $\left\{x_{n}\right\}$ is bounded, there exists $R>0$ such that $\left\{x_{n}\right\} \subset C: B_{R}(0) \cap K$, where $B_{R}(0)=\{x \in X:\|x\| \leq R\}$. Then $C$ is a nonempty closed convex bounded subset of $X$. Let $a_{n}(t)=\left\|t x_{n}+(1-t) p_{1}-p_{2}\right\|$. Then $\lim _{n \rightarrow \infty} a_{n}(0)=$ $\left\|p_{1}-p_{2}\right\|$ and from Lemma 3.1, $\lim _{n \rightarrow \infty} a_{n}(1)=\left\|x_{n}-p_{2}\right\|$ exists. It now remains to prove the lemma for $t \in(0,1)$. Define $U_{n}, W_{n}: C \rightarrow C$ by

$$
\left\{\begin{array}{c}
U_{n} x=P\left(\left(1-\beta_{n}\right) x+\beta_{n} T_{i}\left(P T_{i}\right)^{n-1} x\right) \\
W_{n} x=P\left(\left(1-\alpha_{n}\right) T_{i}\left(P T_{i}\right)^{n-1} x+\alpha_{n} I_{i}\left(P I_{i}\right)^{n-1} U_{n} x\right)
\end{array}, n \geq 1\right.
$$

for all $x \in K$. Then for all $x, y \in K$, we have

$$
\begin{aligned}
\left\|U_{n} x-U_{n} y\right\| & \leq\left\|\begin{array}{c}
P\left(\left(1-\beta_{n}\right) x+\beta_{n} T_{i}\left(P T_{i}\right)^{n-1} x\right) \\
-P\left(\left(1-\beta_{n}\right) y+\beta_{n} T_{i}\left(P T_{i}\right)^{n-1} y\right)
\end{array}\right\| \\
& \leq\left\|\left(1-\beta_{n}\right)(x-y)+\beta_{n}\left(T_{i}\left(P T_{i}\right)^{n-1} x-T_{i}\left(P T_{i}\right)^{n-1} y\right)\right\| \\
& \leq\left(1-\beta_{n}\right)\|x-y\|+\beta_{n}\left(1+l_{n}\right)\left\|I_{i}\left(P I_{i}\right)^{n-1} x-I_{i}\left(P I_{i}\right)^{n-1} y\right\| \\
& \leq\left(1-\beta_{n}\right)\|x-y\|+\beta_{n}\left(1+l_{n}\right)\left(1+k_{n}\right)\|x-y\| \\
& \leq\left(1+l_{n}\right)\left(1+k_{n}\right)\|x-y\|
\end{aligned}
$$

and so

$$
\begin{aligned}
\left\|W_{n} x-W_{n} y\right\| \leq\left\|\begin{array}{c}
P\left(\left(1-\alpha_{n}\right) T_{i}\left(P T_{i}\right)^{n-1} x+\alpha_{n} I_{i}\left(P I_{i}\right)^{n-1} U_{n} x\right) \\
-P\left(\left(1-\alpha_{n}\right) T_{i}\left(P T_{i}\right)^{n-1} y+\alpha_{n} I_{i}\left(P I_{i}\right)^{n-1} U_{n} y\right)
\end{array}\right\| \\
\leq\left\|\begin{array}{c}
\left(1-\alpha_{n}\right)\left(T_{i}\left(P T_{i}\right)^{n-1} x-T_{i}\left(P T_{i}\right)^{n-1} y\right) \\
+\alpha_{n}\left(I_{i}\left(P I_{i}\right)^{n-1} U_{n} x-I_{i}\left(P I_{i}\right)^{n-1} U_{n} y\right)
\end{array}\right\| \\
\leq\left(1-\alpha_{n}\right)\left\|T_{i}\left(P T_{i}\right)^{n-1} x-T_{i}\left(P T_{i}\right)^{n-1} y\right\| \\
\quad+\alpha_{n}\left\|I_{i}\left(P I_{i}\right)^{n-1} U_{n} x-I_{i}\left(P I_{i}\right)^{n-1} U_{n} y\right\| \\
\leq\left(1-\alpha_{n}\right)\left(1+l_{n}\right)\left(1+k_{n}\right)\|x-y\| \\
\quad+\alpha_{n}\left(1+k_{n}\right)\left\|U_{n} x-U_{n} y\right\| \\
\leq\left(1-\alpha_{n}\right)\left(1+l_{n}\right)\left(1+k_{n}\right)\|x-y\| \\
\quad+\alpha_{n}\left(1+k_{n}\right)\left(1+l_{n}\right)\left(1+k_{n}\right)\|x-y\|
\end{aligned}
$$




$$
\begin{aligned}
& \leq\left(1+l_{n}\right)\left(1+k_{n}\right)\left[1-\alpha_{n}+\alpha_{n}\left(1+k_{n}\right)\right]\|x-y\| \\
& \leq\left(1+l_{n}\right)\left(1+k_{n}\right)\left(1+\alpha_{n} k_{n}\right)\|x-y\| \\
& \leq\left(1+l_{n}\right)\left(1+k_{n}\right)^{2}\|x-y\| .
\end{aligned}
$$

Setting

$$
R_{n, m}=W_{n+m-1} W_{n+m-2} \cdots W_{n}, n, m \geq 1,
$$

and

$$
b_{n, m}=\left\|R_{n, m}\left(t x_{n}+(1-t) q_{1}\right)-\left(t R_{n, m} x_{n}+(1-t) R_{n, m} q_{1}\right)\right\| .
$$

From (24) and (25), we have

$$
\left\|R_{n, m} x-R_{n, m} y\right\| \leq L_{n}\|x-y\|
$$

for all $x, y \in C$, where $L_{n}=\prod_{j=n}^{n+m-1}\left(1+l_{j}\right)\left(1+k_{j}\right)^{2}$ and $R_{n, m} x_{n}=x_{n+m}$, $R_{n, m} q=q$ for all $q \in F$. Thus

$$
\begin{aligned}
a_{n}(t) & =\left\|t x_{n}+(1-t) q_{1}-q_{2}\right\| \\
& \leq b_{n, m}+\left\|R_{n, m}\left(t x_{n}+(1-t) q_{1}\right)-q_{2}\right\| \\
& \leq b_{n, m}+L_{n} a_{n}(t) .
\end{aligned}
$$

It follows from (26), (27) and Lemma 2.3 that

$$
b_{n, m} \leq \phi^{-1}\left(\left\|x_{n}-q_{1}\right\|-L_{n}^{-1}\left\|x_{n+m}-q_{1}\right\|\right) .
$$

By Lemma 3.1 and $\lim _{n \rightarrow \infty} L_{n}=1$, we have $\lim _{n, m \rightarrow \infty} b_{n, m}=0$ and so

$$
\lim \sup _{n \rightarrow \infty} a_{n}(t) \leq \lim _{n, m \rightarrow \infty} b_{n, m}+\lim \inf _{n \rightarrow \infty} L_{n} a_{n}(t)=\lim \inf _{n \rightarrow \infty} a_{n}(t) .
$$

This completes the proof.

Finally, we give our weak convergence convergence theorem of the iterative scheme (1). But, we fistly want to draw the attention of the reader towards the following remark.

Remark 4.1. In [14], it is point out that there exist uniformly convex Banach spaces which have neither a Fréchet differentiable norm nor the Opial property but their duals do have the Kadec-Klee property. And the duals of reflexive Banach spaces with Fréchet differentiable norms or the Opial property have the Kadec-Klee property.

Theorem 4.1. Let $X$ be a real uniformly convex Banach space such that its dual $X^{*}$ has the Kadec-Klee property and $K$ be a nonempty closed convex subset of $X$ which is also a nonexpansive retract with retraction $P$. Let $\left\{T_{i}\right\}_{i}^{N}: K \rightarrow X$ be $N$ nonself $I_{i}$-asymptotically nonexpansive mappings with sequences $\left\{l_{n}^{(i)}\right\} \subset[0, \infty)$ such that $\sum_{n=1}^{\infty} l_{n}^{(i)}<\infty$ and $\left\{I_{i}\right\}_{i}^{N}: K \rightarrow X$ be $N$ nonself asymptotically nonexpansive mappings with sequences $\left\{k_{n}^{(i)}\right\} \subset$ 
$[0, \infty)$ such that $\sum_{n=1}^{\infty} k_{n}^{(i)}<\infty$. Let $\left\{\alpha_{n}\right\}$ and $\left\{\beta_{n}\right\}$ be sequences in $[a, 1-a]$ for some $a \in(0,1)$. Suppose that for any given $x_{1} \in K$, the sequence $\left\{x_{n}\right\}$ is generated by (1) and $F=\bigcap_{i=1}^{N} F\left(T_{i}\right) \cap F\left(I_{i}\right) \neq \emptyset$. Then $\left\{x_{n}\right\}$ converges weakly to a common fixed point of $\left\{T_{i}\right\}_{i}^{N}$ and $\left\{I_{i}\right\}_{i}^{N}$.

Proof. Since $\left\{x_{n}\right\}$ is bounded and $X$ is reflexive, there exists a subsequence $\left\{x_{n_{j}}\right\}$ of $\left\{x_{n}\right\}$ which converges weakly to some $q \in K$. Moreover, we have $\lim _{n \rightarrow \infty}\left\|x_{n}-T_{i} x_{n}\right\|=\lim _{n \rightarrow \infty}\left\|x_{n}-I_{i} x_{n}\right\|=0$ for all $i \in J$ by Lemma 3.2 and so $q \in F$ by Lemma 2.4.

Now, we show that $\left\{x_{n}\right\}$ converges weakly to $q$. Suppose that $\left\{x_{n_{k}}\right\}$ is another subsequence of $\left\{x_{n}\right\}$ which converges weakly to some $q^{*} \in K$. By the same method as above, we have $q^{*} \in F$ and $q, q^{*} \in \omega_{w}\left\{x_{n}\right\}$. By Lemma 4.1 ,

$$
\lim _{n \rightarrow \infty}\left\|t x_{n}+(1-t) q-q^{*}\right\|
$$

exists for all $t \in[0,1]$ and so $q=q^{*}$ by Lemma 2.5. Therefore, the sequence $\left\{x_{n}\right\}$ converges weakly to $q$. This completes the proof.

Remark 4.2. (1) Our theorems not only improve and generalize important related results of the previously known results in this area, but also even in the case of $I_{i}=E$ for all $1 \leq i \leq N, I_{i}$ and $T_{i}$ self-map of $K$ for all $1 \leq i \leq N, \alpha_{n}=1$ or $\beta_{n}=0$ also all are new.

(2) If the error terms are added in (1) and assumed to be bounded, then the results of this paper still hold.

\section{REFERENCES}

[1] K. Goebel, W.A. Kirk, A fixed point theorem for asymptotically nonexpansive mappings, Proc. Amer. Math. Soc., 35 (1972), 171-174.

[2] R.P. Agarwal, Donal O'Regan and D.R. Sahu, Iterative construction of fixed points of nearly asymptotically nonexpansive mappings, J.Nonliear Convex. Anal. 8 (1) (2007), 61-79.

[3] S.H. Khan, M. Abbas, Approximating common fixed points of nearly asymptotically nonexpansive mappings, Anal. Theory Appl., Vol. 27, No. 1 (2011), 76-91.

[4] S. Temir, On the convergence theorems of implicit iteration process for a finite family of I-asymptotically nonexpansive mappings, J. Comput. Appl. Math. 225 (2009), 398-405.

[5] S. Temir, O. Gul, Convergence theorem for I-asymptotically quasi-nonexpansive mapping in Hilbert space, J. Math. Anal. Appl. 329 (2007), 759-765.

[6] L. Yang, X. Xie, Weak and strong convergence theorems for a finite family of Iasymptotically nonexpansive mappings, Appl. Math. Comput. 216 (2010), 1057-1064.

[7] F. Gu, Some convergence theorems of non-implicit iteration process with errors for a finite families of I-asymptotically nonexpansive mappings, Appl. Math. Comput. 216 (2010), 161-172. 
[8] K.K. Tan and H.K. Xu, Approximating fixed points of nonexpansive mappings by the Ishikawa iteration process, Journal of Mathematical Analysis and Applications, vol. 178, no. 2 (1993), pp. 301-308.

[9] J. Schu, Weak and strong convergence to fixed points of asymptotically nonexpansive mappings, Bull. Austral. Math. Soc. 43 (1991), 153-159.

[10] M.O. Osilike, A. Udomene, Demiclosedness principle and convergence theorems for strictly pseudocontractive mappings of Browder-Petryshyn type, J. Math. Anal. Appl. 256 (2001), 431-445.

[11] H.F. Senter, W.G. Dotson, Approximating fixed points of nonexpansive mappings, Proc. Amer. Math. Soc., 44 (1974), 375-380.

[12] S.H. Khan, H. Fukharuddin, Weak and strong convergence of a scheme with errors for two nonexpansive mappings, Nonlinear Anal. 61 (2005), 1295-1301.

[13] J.G. Falset, W. Kaczor, T. Kuczumow, S. Reich, Weak convergence theorems for asymptotically nonexpansive mappings and semigroups, Nonlinear Anal. 43 (2001), 377-401.

[14] W. Kaczor, Weak convergence of almost orbits of asymptotically nonexpansive commutative semigroups, J. Math. Anal. Appl. 272 (2002), 565-574.

[15] C.E. Chidume, E.U. Ofoedu, H. Zegeye, Strong and weak convergence theorems for asymptotically nonexpansive mappings, J. Math. Anal. Appl., 280 (2003), 364-374.

\section{BIROL GÜNDÜZ}

Department of Mathematics

Faculty of Science And Art

ERZINCAN UNIVERSITY

24000, ERZINCAN

TURKEY

E-mail address: birolgndz@gmail.com

Sezgin Akbulut

Department of Mathematics

Faculty of Science

Ataturk University

25240, ERZURUM

TURKEY

E-mail address: sezginakbulut@atauni.edu.tr 